\title{
Reference free Chi 3 dispersion measurements in planar tantalum pentoxide waveguides
}

\author{
Ruiqi Y. Chen ${ }^{\mathrm{a}}$, Martin D. B. Charlton ${ }^{\mathrm{a}}$, Pavlos G. Lagoudakis ${ }^{\mathrm{b}}$ \\ ${ }^{a}$ School of Electronics and Computer Science, University of Southampton, SO17 1BJ, U.K.; \\ ${ }^{\mathrm{b}}$ School of Physics and Astronomy, University of Southampton, SO17 1BJ, U.K.
}

\begin{abstract}
We utilize analysis of third harmonic generation under femtosecond pulsed excitation as a reference free measurement method for third order nonlinear susceptibility ( $\chi^{(3)}$ or "Chi 3") of planar waveguides. We investigate $\chi^{(3)}$ dispersion in planar $\mathrm{Ta}_{2} \mathrm{O}_{5}$ waveguides at wavelengths either side of the telecoms window, obtaining a nonlinear coefficient of $2 \times 10^{-13}$ esu, at $1550 \mathrm{~nm}$. Our study indicates that $\chi^{(3)}$ increases within the measured wavelength range due to a threephoton resonance of $\mathrm{Ta}_{2} \mathrm{O}_{5}$ electrons, revealing the potential of this material system in high speed integrated nonlinear optical switches for the telecommunications spectral window.
\end{abstract}

Keywords: waveguides, chi 3, dispersion

\section{INTRODUCTION}

Nonlinear susceptibilities are known to be useful in studying physical properties of dielectrics and therefore important for applications[1] such as optical interferometers[2], supercontinuum generation (SCG)[3, 4], wavelength division multiplexing (WDM) for optical communications[2, 5], optical parametric amplifiers[6], and are widely used in both laboratory and commercial laser systems (e.g. Coherent Inc.). There is interest in engineering compact devices to operate with lower power solid state pump sources such as vertical-external-cavity surface-emitting semiconductor laser (VECSEL) $[7,8]$ in order to move applications out of the laboratory. Since the second-order nonlinear susceptibility $\left(\chi^{(2)}\right)$ does not exist for materials with centrosymmetric lattice such as silica and $\mathrm{Ta}_{2} \mathrm{O}_{5}$, nonlinear phenomena occurring within these materials are governed by the third-order susceptibility $\left(\chi^{(3)}\right)[9,10]$. A number of techniques for measuring $\chi^{(3)}$ of materials have been reported in literature. These include:

1) Self-phase modulation (SPM)[11]. This is based on measurement of SPM induced spectral broadening of femtosecond pulses. In this case pulse duration is a critical parameter, hence group velocity dispersion (GVD) must be taken into consideration as the pulse propagates along the guide, and time-of-flight or autocorrelation methods are required to measure the pulse duration. Nonlinear coefficients of the guiding medium can then be derived at the wavelength of pulse duration measurement.

2) Four-wave mixing (FWM)[12-15] is one of the most common method for measurement of $\chi^{(3)}$, either by measuring the two-photon absorption coefficient, or by comparison of the time resolved signal intensities of degenerate FWM (DFWM).

3) The Z-scan technique[16, 17] is a well accepted method for measuring both real and imagery $\chi^{(3)}$ coefficients. The technique is used to examine mostly thin film layers, where $\chi^{(3)}$ is derived from the differences between input and output signals as the sample position changes along the propagation direction (i.e. z-direction).

4) The Maker fringes method[18-20] has been used to examine thin film layers on a known substrate (e.g. fused silica) and is similar to the Z-scan method. However, instead of moving the sample in the z-direction, the sample is rotated on a plate to adjust phase matching conditions and the nonlinear coefficient is extracted by examining fringes which occurs through interference of the detected third-harmonic intensity in a Maker interferometer. 
5) Third harmonic generation (THG)[21-23] has also been used to determine $\chi^{(3)}$ (mainly for single crystal structures). Previous experiments measured the intensity/polarization field of THG in single/sapphire crystals and optical fibers to obtain $\chi^{(3)}$ values. With this method a known reference material is required (most commonly fused silica).

Of the above listed methods, THG measurement is one of the simplest possible methods for obtaining $\chi^{(3)}$ since it is a purely electronic and coherent process, with no ambiguity in the physical mechanism. This is in contrast to other processes such as degenerate four-wave mixing and optical Kerr effect[23], where other competing nonlinear effects complicate the experimental analysis. THG has been observed in several materials including: BIBO (Bismuth triborate, $\left.\mathrm{BiB}_{3} \mathrm{O}_{6}\right)$ [24], thin sapphire crystals[25], fused silica[26], carbon nanotubes[27], and other types of single crystals[28].

Tantalum pentoxide $\left(\mathrm{Ta}_{2} \mathrm{O}_{5}\right)$ planar waveguides have recently been shown to possess unusually large third order nonlinear susceptibilities ( $\chi^{(3)}$ or "Chi 3") and nonlinear Kerr coefficient $\left(n_{2}\right)$ [11]. Other materials such as silicon or chalcogenide $\left(A s_{2} S_{3}\right)$ glass have also shown very high $\chi^{(3)}$ or $n_{2}$, but silicon suffers from high intrinsic normal dispersion and free carrier (FC) creation losses[29, 30] and chalcogenide glass also has high normal dispersion and relatively low damage threshold $\left(9 \mathrm{GW} / \mathrm{cm}^{2}[31,32]\right)$ compared to $\mathrm{Ta}_{2} \mathrm{O}_{5}$ [3] used as a high $k$-dielectric for silicon heterojunction bipolar transistors ( $\mathrm{Si}-\mathrm{HBTs}$ ). $\mathrm{Ta}_{2} \mathrm{O}_{5}$ is a well known material to the microelectronics industry[33] with well known processing technologies, and forms a strong candidate for potential applications in nonlinear integrated optics[34-38], such as supercontinuum generation (SCG) [3, 39, 40], third harmonic generation (THG) spectroscopy[4143], compact optical parametric amplifiers (OPAs) and tunable light sources as well as fluorescence detection[44-46].

We report the experimental demonstration of THG in planar $\mathrm{Ta}_{2} \mathrm{O}_{5}$ waveguides and perform analysis of frequency tripling effects under a range of pump conditions, and together with detailed calculations of $\chi^{(3)}$ through THG based on the work of Meredith et al.[22] and Kobayashi et al.[23]. Our revised method does not require a reference material (e.g. fused silica), and works even in the presence of other competing nonlinear effects such as self-phase modulation (SPM), Raman effects, and four-wave mixing (FWM) (including standard degenerate four-wave mixing (DFWM)). We utilize the relationship between $\chi^{(3)}$ and $n_{2}$ in solid semiconductors (described in the work of Mohanta and Choudhury[14]) to compare the derived values of our results with the first reported measurement of the nonlinear coefficient $\left(n_{2}\right)$ of $\mathrm{Ta}_{2} \mathrm{O}_{5}$ planar waveguides by Tai et al.[11] whose measurement was based on analysis of self-phase modulation at a fixed wavelength of $800 \mathrm{~nm}$.

In this paper, we present the $\chi^{(3)}$ dispersion in $\mathrm{Ta}_{2} \mathrm{O}_{5}$ for the first time. $\chi^{(3)}$ dispersion has been studied in material systems such as poly(dihexylsilane) (PDHS) films[47], molecules[48], and $\mathrm{TeO}_{2}$ glass films[18] by analyzing the Kramers-Kronig dispersion relations, absorption spectrum, and THG respectively. Our measurement of $\chi^{(3)}$ is extended over a range of wavelength from $1470-1610 \mathrm{~nm}$, enabling us to obtain the $\chi^{(3)}$ dispersion in $\mathrm{Ta}_{2} \mathrm{O}_{5}$ material system.

\section{EXPERIMENTS AND RESULTS}

\subsection{Device Fabrication and Testing}

$\mathrm{Ta}_{2} \mathrm{O}_{5}$ waveguides samples were grown on a silicon substrate by RF (radio frequency) sputtering, optical lithography, and plasma etching[3]. Figure 1 shows a cross-sectional SEM (scanning electron microscope) image of the waveguide sample analyzed. Robustness tests have previously shown that there is no change in waveguide loss or UV induced damage with up to $1 \mathrm{~W}$ of continuous wave (CW) $455 \mathrm{~nm}$ light focused into the guide, and no waveguide facet damage for $800 \mathrm{~nm} 250 \mathrm{fs}$ pulses with energies up to $2 \mu \mathrm{J}$ from a $250 \mathrm{kHz}$ Ti:Sapphire regenerative amplifier[3, 40]. Before commencing this new analysis of nonlinear effects in the guides, further tests for laser induced damage were performed on a test guide. This showed that there was no damage for $250 \mathrm{fs}$ pulses with energies up to the maximum power output $(12 \mathrm{~nJ} /$ pulse or $3 \mathrm{~mW}$ average power) over the entire $900-1650 \mathrm{~nm}$ tuning range of our OPA, which was sufficient for this experiment. 

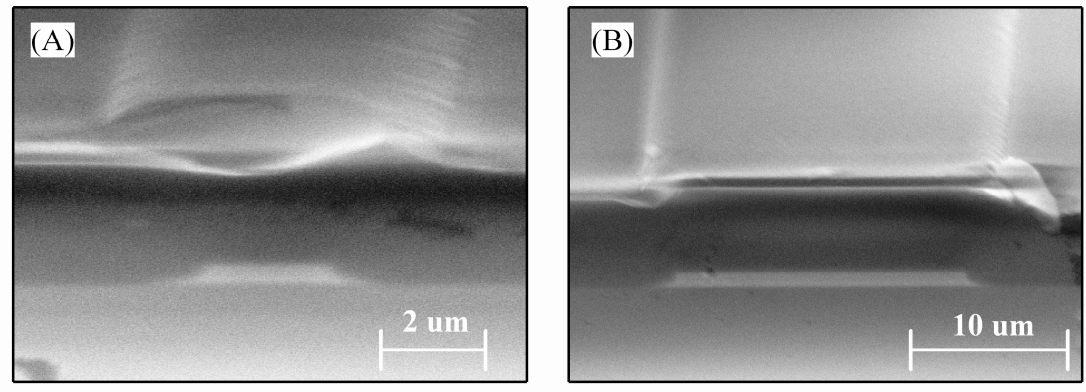

Fig. 1. SEM images of $750 \mathrm{~nm}$ high tantalum pentoxide waveguide samples. Sample A: 2.5 micron wide (Left); Sample B: 18 micron wide (Right).

\subsection{Experimental Setup}

Figure 2 shows the experimental setup used for $\chi^{(3)}$ measurement. The fundamental pump beam is provided by an OPA pumped by a mode-locked ultrafast Ti-Sapphire amplifier. The OPA provides tunable infra-red (IR) pulses (250 fs duration) over range 900 to $1600 \mathrm{~nm}$ (limited by the detection equipment). A telescope is used to reduce the pump spot size for improved coupling to the guides. A wave plate and a linear polarizer are used as a power attenuator. This allows input power to be adjusted without introducing significant distortions into the beam or affecting the coupling conditions. However, due to the high power pump and low damage threshold of the wave plate, we remove the wave plate and rotate polarizer as the power attenuator, and correct the output power correspondingly. A short focal length aspheric lens (which provides a near transform limited spot) is used for input coupling to the guides and output from the end facets of the guides are collected and collimated by a 10X microscope objective. For analysis of IR spectra, the output from the guide is fiber coupled to an Ocean Optics NIR512 IR spectrometer. For analysis of visible spectra, KG5 and SPF650 filters are used to remove the residual pump before fiber coupling to a visible spectrometer (Ocean Optics HR2000). Additional lenses and removable beam splitters image the waveguide output facet onto separate visible and IR CCD cameras placed after the microscope objective. Images from these cameras are used to optimize pump input coupling and to identify the mode number of the coupled pump beam and the generated THG modes.

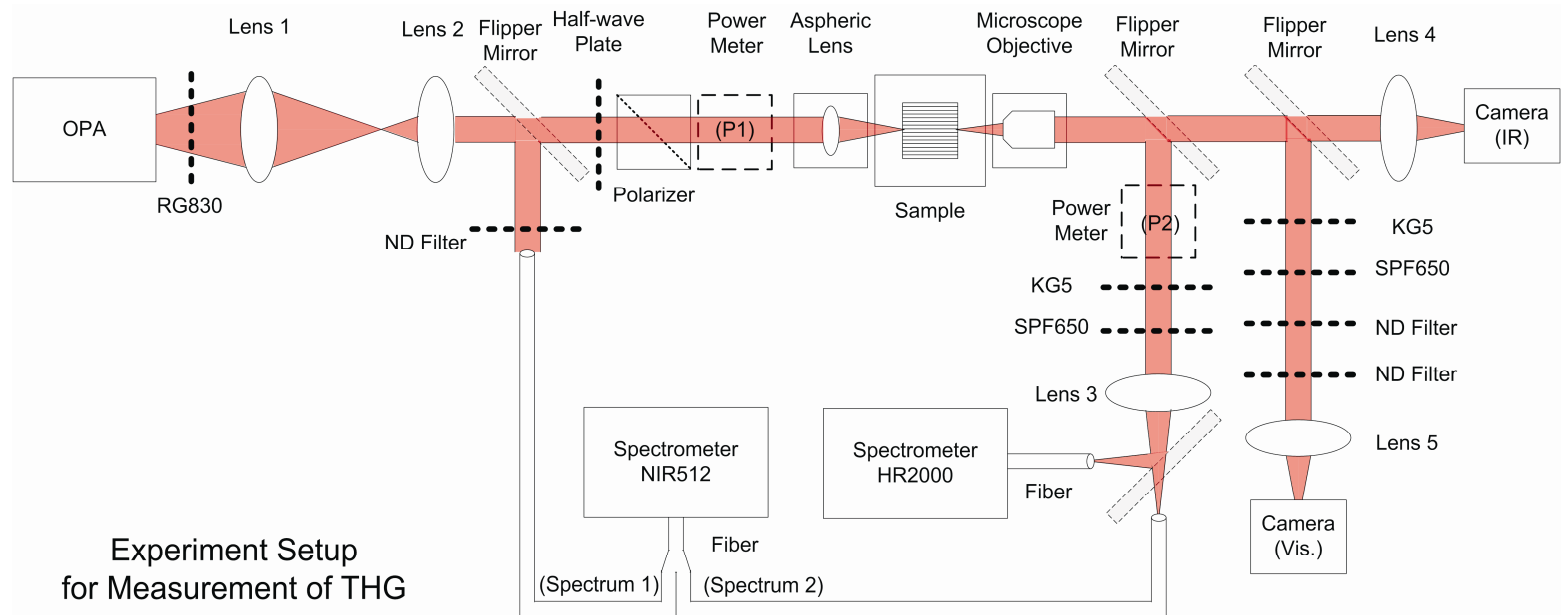

Figure 2: Experimental setup for the third order nonlinear susceptibility (Dashed items are removable/replaceable according to different wavelengths, equipments, or experimental requirements). The output beam from OPA is firstly coupled into a telescope to reduce the spot size for improved coupling; the input coupling power is adjusted by an attenuator (a polarizer and half-wave plate); aspheric lens is used to provide a near transform limited spot to couple into the waveguide; output signals collected by a microscope objective are focused onto different spectrometers and cameras for analysis with appropriate filters applied. 


\subsection{Experiment}

Silicon dioxide $\left(\mathrm{SiO}_{2}\right.$ ) clad $\mathrm{Ta}_{2} \mathrm{O}_{5}$ planar rib waveguides with core thickness of $750 \mathrm{~nm}$, and widths $2.5 \mu m$ (Guide A) and $18 \mu \mathrm{m}$ (Guide B) were analyzed. The pump was tuned over wavelength range $1470-1610 \mathrm{~nm}$, and spectral measurements made at average pump powers in the range $0.5-2.0 \mathrm{~mW}$ with steps of $0.5 \mathrm{~mW}$ (measured by power meter P1 placed between polarizer and input coupling optics). Frequency tripling was observed over the wavelength range 489 $-538 \mathrm{~nm}$. Figure 3a shows an example set of the normalized IR pulse spectrum at $1550 \mathrm{~nm}$ pump wavelength with the pump mode profile, corresponding THG output spectrum (normalized) and THG mode profile. The two peaks appeared at the similar power levels and the additional THG peak in figure $3 \mathrm{a}$ clearly shows that there are several competing nonlinear effects occurring within the waveguide for pulse energies of $8 \mathrm{~nJ}(2 \mathrm{~mW}$ average power) for $250 \mathrm{fs}$ pulse width at $1550 \mathrm{~nm}$. Figure $3 \mathrm{~b}$ shows the magnified IR pulse spectrum (normalized) and the corresponding residual pump spectrum (normalized) in log scale.
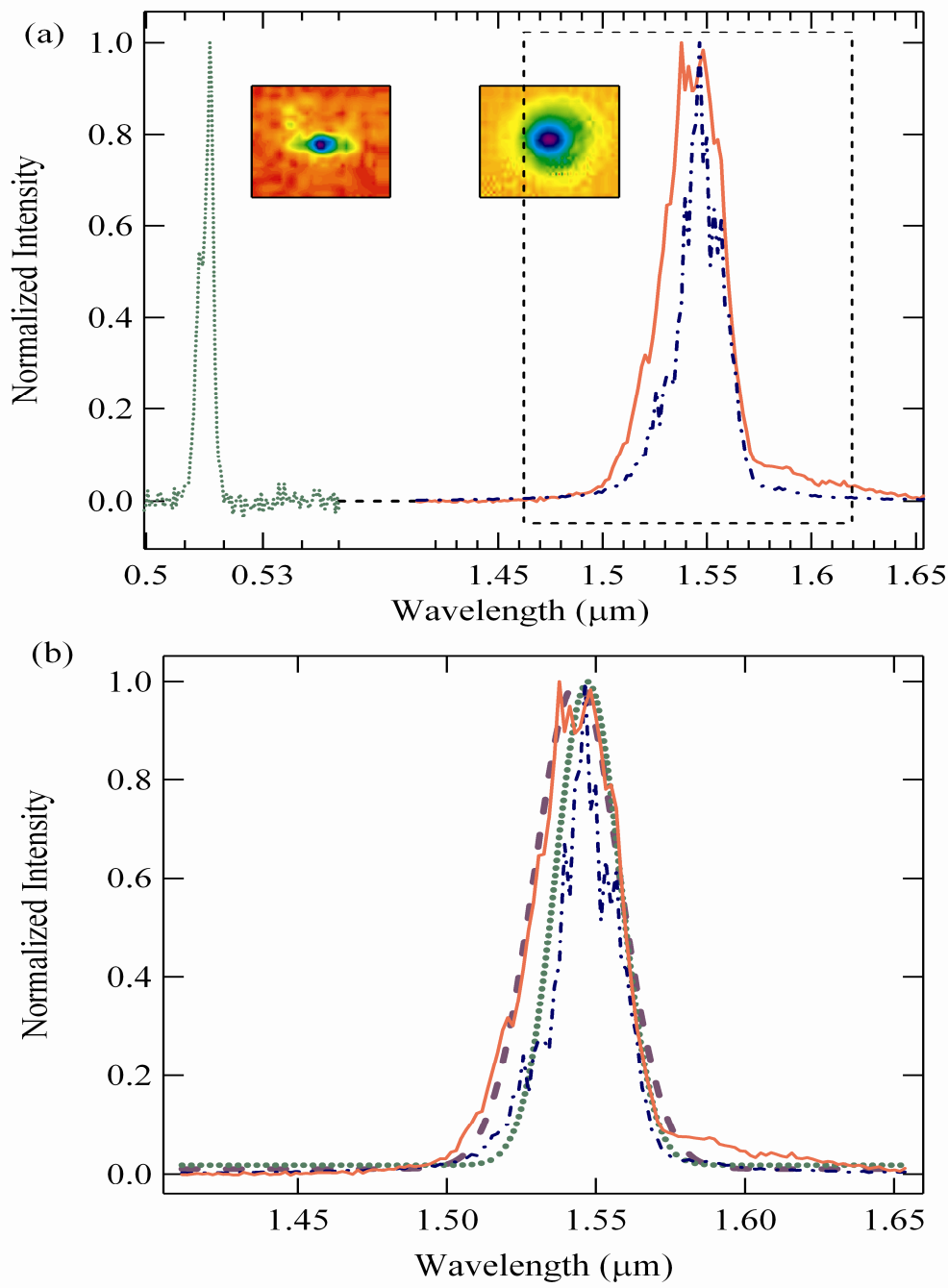

Figure 3: (a) IR (solid red line) and THG output (dotted green line) spectra of the guide A at $1550 \mathrm{~nm}$ pump (dashed blue line) wavelength with average power of $2 \mathrm{~mW}(8 \mathrm{~nJ})$. Insets: (left) mode profile of THG output, (right) mode profile of IR output. (b) IR (solid red line) and its pump (dashed blue line) spectra with the respective normalized Gaussian fits of the un-broadened input pump spectrum (dotted green line) and the respective residual broadened pump in IR output spectrum (broken purple line). 


\subsection{Theory}

$\chi^{(3)}$ is related to the nonlinear Kerr effect which describes how the refractive index of a nonlinear material changes with the incident light intensity[49]:

$$
n=n_{0}+n_{2} \cdot I
$$

where for solid semiconductors, the nonlinear refractive index $\left(n_{2}\right)$ can be written as[14]:

$$
n_{2}=\frac{16 \pi^{2}}{c n_{0}{ }^{2}}\left|\chi^{(3)}\right|
$$

In order to measure $\chi^{(3)}$ of the material, the following mathematical relations linking THG field intensities and $\chi^{(3)}$ can be used:

Under the perfect phase matching condition, the electric fields of second harmonic generation (SHG) and a pump beam propagating along the $\mathrm{z}$-direction is given by[50]:

$$
\frac{d}{d z} E(z, 2 \omega)=i \kappa \cdot e^{-i \Delta k \cdot z} \cdot E^{2}(z, \omega)
$$

where $\quad \kappa=\frac{\omega d_{\text {eff }}}{n_{0} c} \quad$ with $\quad d_{\text {eff }}=\varepsilon_{0} \cdot \chi^{(2)}$, and $\Delta k=k(2 \omega)-2 k(\omega)$ is the wave number difference, $k=\frac{2 \pi}{\lambda_{\text {wg }}}=\frac{2 \pi}{\lambda_{\text {pump }} / n_{0}}=\frac{2 \pi \cdot n_{0}}{\lambda_{\text {pump }}}$ is the wave number inside the waveguide.

hence, integrating both sides of equation (3) gives:

$$
|E(2 \omega)|^{2}=\left(\frac{\omega d_{e f f}}{n_{0} c}\right)^{2} \cdot|E(\omega)|^{4} \cdot \frac{1}{\Delta k^{2}}
$$

Applying the same phase-matching condition for THG, the electric fields must satisfy the relations:

$$
|E(3 \omega)|^{2}=\left(\frac{\omega d_{\text {eff }}}{n_{0} c}\right)^{2} \cdot|E(\omega)|^{6} \cdot \frac{1}{\Delta k^{2}}
$$

where in this case, $d_{\text {eff }}=\chi^{(3)}$ for the third harmonic generation[51].

The intensity per unit area is given by: $\frac{\mid \text { Power }\left.\right|^{2}}{\text { Area }}=I=\frac{c n_{0} \varepsilon_{0}}{2} E \cdot E^{*}=\frac{c n_{0} \varepsilon_{0}}{2}|E|^{2}$, therefore, the relationship between THG intensity and $\chi^{(3)}$ under the perfect phase-matching conditions can be written as:

$$
I(3 \omega)=\frac{4 \omega^{2}}{\left(c n_{0}\right)^{4} \cdot \Delta k^{2} \cdot \varepsilon_{0}{ }^{2}} \cdot\left|\chi^{(3)}\right|^{2} \cdot I(\omega)^{3}
$$

where $n_{0}=n_{\text {eff }}, \omega=\frac{2 \pi \cdot c}{\lambda_{\text {pump }}}, \Delta k=k(3 \omega)-3 k(\omega)$, and $\chi^{(3)}$ can then be derived:

$$
\left|\chi^{(3)}\right|=\sqrt{I(3 \omega) \cdot \frac{\left(c n_{0}\right)^{4} \cdot \Delta k^{2} \cdot \varepsilon_{0}{ }^{2}}{4 \omega^{2} \cdot I(\omega)^{3}}}
$$


Although only the optimized phase-matching condition is considered in this paper for simplicity and easy understanding, it is worth mentioning that the complete form of equation (4) including phase-mismatch can be written[52]:

$$
I(3 \omega)=\frac{\omega^{2}}{\left(c n_{0}\right)^{4} \cdot \varepsilon_{0}{ }^{2}} \cdot\left|\chi^{(3)}\right|^{2} \cdot I(\omega)^{3} \cdot l^{2} \cdot\left[\frac{\sin (\Delta k \cdot l / 2)}{(\Delta k \cdot l / 2)}\right]^{2}
$$

where $l$ is the effective propagation length along the guide, and $\left[\frac{\sin (\Delta k \cdot l / 2)}{(\Delta k \cdot l / 2)}\right]^{2}$ is the phase-matching term.

Therefore, values and parameters required in order to calculate $\chi^{(3)}$ from equation (7) include: intensity of the coupled fundamental pump mode (measured at the beginning the waveguide), intensity of the generated THG (measured at the output of the waveguide), cross-sectional area of the coupled pump mode, wave numbers (k-vectors) for the coupled fundamental pump mode, and the generated THG modes.

\subsection{Methodology for experimental determination of Chi 3}

The effective mode indices, mode spot size (1/e widths), and wave vectors $(\mathrm{k})$ at the test pump wavelength can be are determined numerically by applying finite element analysis (RSoft CAD Suite software) to the waveguide design. Our model takes into account material dispersion as detailed in $[53,54]$. Figure 4 shows the calculated effective indices according to the mode profiles shown in figure $3 \mathrm{a}$, and the corresponding wave number differences for both guide $\mathrm{A}$ and guide B. The numerical accuracy of the simulation solutions of effective mode index was set to the 10th decimal place, providing more than sufficient accuracy to calculate the derivatives for this analysis.

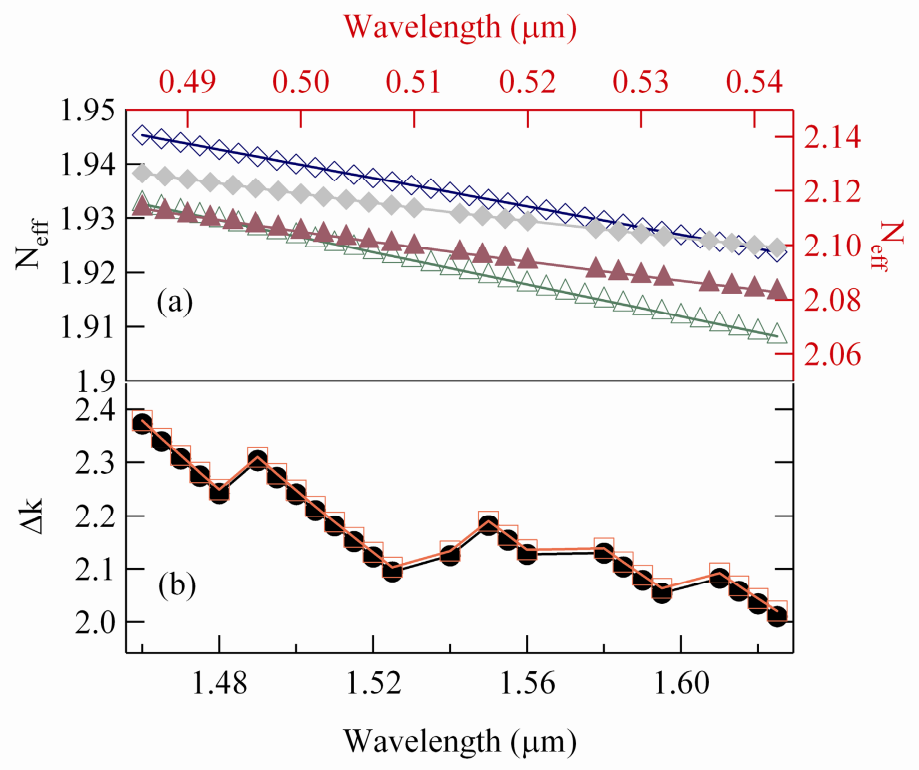

Figure 4: (a) Effective refractive indices of pump beam and THG signal for sample A with open triangle (green) and solid triangle (purple), respectively; effective indices of pump beam and THG signal for sample B with open diamond (blue) and solid diamond (grey), respectively. (b) The resulting wave number differences with solid circles (black) for sample A, and open square (red) for sample B.

To determine the true output intensity of the THG signal from the fiber coupled spectrometer measurements, we must first calibrate the spectrometer to take into account efficiency of collection of light by the fiber in the back focal plane of the output objective. To do this, a continuous wave (CW) He-Ne laser was directed onto a $10 \mu m$ pin hole. Light emerging from the rear of the pin hole was then collected using exactly the same objective arrangement as used for the THG experiments. The integrated area of the spectrum measured on the spectrometer is then normalized with a reading taken from power meter (placed in the same position behind the objective). In this arrangement, the pin hole effectively mimics the diffractive properties of the waveguide, but excludes inaccuracies which would otherwise be introduced by 
the presence of nonlinear effects or loss in the guide. As the spectrometer is pre-calibrated over all wavelengths, a single wavelength calibration measurement is then sufficient to normalize the experimental data at other wavelengths.

In figure $3 b$, we see that the pump has been significantly broadened. This is due to a combination of self-phase modulation and four-wave mixing. In order to calculate $\chi^{(3)}$ precisely, independent from these effects, the power contributed by fundamental residual pump (coupled at the waveguide input facet) within the output has to be separated from the broadened spectral data (measured behind the objective at the output facet).

At experimental power levels (up to $2 \mathrm{~mW}$ ) a single well defined Gaussian peak is observed at visible wavelengths confirming that only the fundamental pump contribution to the broadened signal observed at IR wavelengths is responsible for THG conversion to the visible. Side peaks in the IR signal induced by other nonlinear effects (such as XPM and FWM) do not therefore contribute power to the THG process (otherwise multiple peaks would be observed in the visible) and so power associated with these side peaks must be removed from the calculations.

Gaussian curve fitting techniques are used to determine the exact make up of contributory components in the observed broadened IR spectra (measured by the same IR spectrometer after filtering out the visible output). In the case of simple SPM induced at low pump power levels, a single Gaussian curve can be used to fit the resultant broadened IR spectra. In the case of XPM and SPM induced at higher pump power levels, three or more Gaussians must be used to fit the broadened IR spectra (exact number depends on the pump power level and the consequent amount of broadening). Conversely the curve fitting analysis allows positive identification of exactly which non linear process contributes to the broadened signal.

For simplicity, and by way of example figure 3 illustrates this process for the case where the fundamental IR pump is broadened by SPM only and hence can be fitted by a single Gaussian curve. Once curve fitting is completed, the power contribution to the THG process is given by the broadened residual pump signal corresponding to the highest amplitude Gaussian, which will itself be broadened by process of SPM. We then normalize the intensities of the Gaussian fit to the pump spectra (measured by the IR spectrometer before coupling into the waveguides) $S_{1}$ (dotted red line in Fig. $3 \mathrm{~b}$ ) and the corresponding Gaussian fit to the residual pump contribution in the output spectra (measured by the same IR spectrometer after filtering out the visible output) $S_{2}$ (solid black line in Fig. 3b) to the same level. We then calculate the integral of the areas of the respective spectra for each input power, i.e. $A_{1}$ and $A_{2}$ are the corresponding integrated areas of $S_{1}$ and $S_{2}$ shown in Fig. 3b, respectively.

$A_{1}=\int S_{1}(\lambda) d \lambda($ after normalization)

$A_{2}=\int S_{2}(\lambda) d \lambda$ (after normalization)

The ratio of $A_{1}$ and $A_{2}$ then indicates how much pump power actually contributes to THG $\left(P_{T H G}\right)$.

Hence by analysing the emergent residual pump spectra using the Gaussian fitting technique we can de-convolve the proportion of coupled pump power which contributes to other competing nonlinear effects (and thus do not contribute to THG) and the proportion of coupled pump power which does contribute directly to THG. In practice power measurements are taken from power meter readings ( $P_{2}$ in Fig. 2) placed behind the objective (output coupling losses can be assumed to be negligible).

$R=\frac{A_{1}}{A_{2}} \& P_{T H G}=\frac{A_{1}}{A_{2}} P_{2}$

In order to remove ambiguities associated with waveguide input coupling efficiency, the actual coupled pump power at the start of the waveguide was determined using known waveguide loss values to scale the obtained output power values from the output $\left(P_{T H G}\right)$. The waveguide loss was measured by cutback method to be $-8.5 \mathrm{~dB} \pm 10 \%$ for sample $\mathrm{A}$ and $4.5 \mathrm{~dB} \pm 10 \%$ for sample $\mathrm{B}$ using identical waveguide samples from the neighbouring areas of the same wafer. 
Inserting the appropriate measured experimental and theoretically derived parameters into equation (7), we obtain the $\chi^{(3)}$ values of $\mathrm{Ta}_{2} \mathrm{O}_{5}$ planar waveguides. Before calculating $\chi^{(3)}$ values, we must take the following into considerations:

1) The analysis must be performed under perfect phase matching conditions (otherwise the value of THG intensity will be an underestimate). In reality, phase matching may not necessarily be optimal, in which case the derived $\chi^{(3)}$ values will be underestimated. We can ensure that phase matching is optimal (in accordance with Equ. 8) by tuning the pump laser wavelength to maximize the intensity of the THG signal.

Theoretical modelling (figure 4) was used in combination with analysis of the image of the coupled mode spot shape (to identify the actual coupled mode number) in order to examine the phase difference between pump and THG signals for different sized guides. This revealed that the wave number difference $(\Delta k)$ between pump and THG does not actually change when the pump is coupled into guides of different widths. This is a clear indication of a self-selective process inside the waveguides, which (provide the guide supports a sufficient number of modes) seems to always find the same (optimal) phase matching conditions irrespective of waveguide width.

2) We note from Fig. $3 b$ that the pump signal is broadened considerably during its transit along the guide, generating strong side peaks due to four-wave mixing. These side peaks could themselves cause additional THG peaks giving rise to triplets of peaks in the visible, in which case absolute power measurement and calculation of relative pump power contribution becomes more complex. We therefore ensure that only power associate with the fundamental mode of the incident pump beam contributes to the generation of a single THG peak by performing the analysis below the power threshold level at which the THG signal becomes split into a triplet.

3) We assume that (as measured previously) the loss of all the waveguides is $-8.5 \mathrm{~dB}$ with $10 \%$ error rate independent of width.

4) Output coupling can be assumed to be perfect since the microscope objective is extremely close to the output facet, such that all the output light is well coupled into the objective. All optical components are AR (anti-reflection) coated specifically for IR wavelengths. There will be a small error in calculation of the visible THG signal power due to coupling loss in the output collection optics. The waveguide losses have $10 \%$ error as indicated from previous measurements. These two factors could introduce a combined error of $\approx \pm 1.57761 \times 10^{-13}$ esu to $\chi^{(3)}$ calculations, which lead to $\approx \pm 2.27532 \times 10^{-19} \mathrm{~m}^{2} / W$ variation of the Kerr nonlinear coefficient $\left(n_{2}\right)$.

\subsection{Chi 3 measurement results}

To further reduce possible measurement errors, the full analysis was performed at two average pump power levels (1.5 $\mathrm{mW}$ and $2 \mathrm{~mW}$ ). Table 1 shows the calculated nonlinear coefficients of each pump wavelength used in the corresponding waveguide for the above average pump power levels. The values derived are corrected by taking into account the polarization changes during the experiment.

Table 1: Calculated third-order nonlinear susceptibility of tantalum pentoxide waveguide samples

\begin{tabular}{|ccc|}
\hline & \multicolumn{2}{c|}{ Guide A $(2.5 \mu m$ wide $) \times 10^{-13}$ esu } \\
$\lambda_{\text {pump }}$ & $\underline{1.5 \mathrm{~mW}}$ & $\underline{2 \mathrm{~mW}}$ \\
$1470 \mathrm{~nm}$ & $0.98 \pm 0.21$ & $1.42 \pm 0.21$ \\
$1500 \mathrm{~nm}$ & $0.80 \pm 0.23$ & $1.02 \pm 0.23$ \\
$1550 \mathrm{~nm}$ & $2.13 \pm 0.57$ & $1.87 \pm 0.57$ \\
$1610 \mathrm{~nm}$ & $4.75 \pm 1.58$ & $5.74 \pm 1.58$ \\
& Guide B $(18 \mu m$ wide $) \times 10^{-13} \mathrm{esu}$ \\
$\frac{1.5 \mathrm{~mW}}{149 \mathrm{nmp}}$ & $\underline{2 \mathrm{~mW}}$ \\
$1555 \mathrm{~nm}$ & $4.23 \pm 0.90$ & $0.96 \pm 0.21$ \\
$1610 \mathrm{~nm}$ & $4.33 \pm 1.26$ & $4.75 \pm 0.90$ \\
& & $6.00 \pm 1.26$ \\
\hline
\end{tabular}




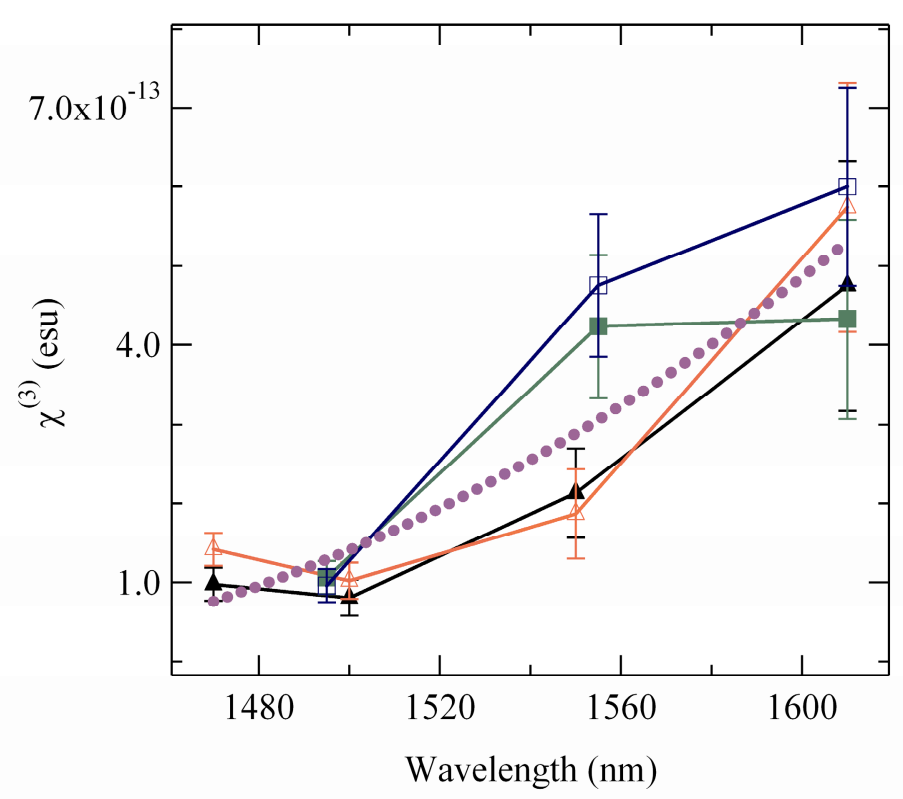

Figure 5: Calculated third-order nonlinear susceptibility of tantalum pentoxide waveguide samples. Sample A: solid triangle (black) for $1.5 \mathrm{~mW}$ and open triangle (red) for $2 \mathrm{~mW}$ pump power; Sample B: solid square (green) for $1.5 \mathrm{~mW}$ and open square (blue) for $2 \mathrm{~mW}$ pump power. Purple dotted line indicates the trend of third-order nonlinear susceptibility changes with wavelength.

The average value for $\chi^{(3)}$ over the wavelength range 1470 to $1610 \mathrm{~nm}$ is: $\chi^{(3)} \approx(2.673 \pm 1.578) \times 10^{-13}$ esu. Hence, the average value of the nonlinear Kerr coefficient is: $n_{2} \approx(3.773 \pm 2.275) \times 10^{-19} \mathrm{~m}^{2} / W=(3.773 \pm 2.275) \times 10^{-15}$ $\mathrm{cm}^{2} / W$. This value is within the acceptance error range with the previously measured value at $800 \mathrm{~nm}\left(7.23 \times 10^{-19}\right.$ $\mathrm{m}^{2} / W$ ) from SPM analysis[11]. It also shows that the value of nonlinear susceptibility of $\mathrm{Ta}_{2} \mathrm{O}_{5}$ is, as expected, in between that of BK7 silica glasses $\left(\chi^{(3)} \approx 2.0 \times 10^{-14} \mathrm{esu}\right)$ and titanium dioxides $\left(\mathrm{TiO}_{2}\right)\left(\chi^{(3)} \approx 1.5 \times 10^{-12} \mathrm{esu}\right)[10]$.

A key advantage of this method of measurement of $\chi^{(3)}$ is that the THG output is only dependent on $\chi^{(3)}$ of the material. By analyzing the spectra and normalizing the fundamental pump contribution at the output as described above, we can precisely calculate $\chi^{(3)}$ with little difficulty in identifying and removing potential contributions from competing effects such as FWM and SPM.

Our multi-wavelength analysis also allows analysis of $\chi^{(3)}$ dispersion, revealing a trend for the $\chi^{(3)}$ value of $\mathrm{Ta}_{2} \mathrm{O}_{5}$ to increase nolinearly with wavelength (figure 5). $\chi^{(3)}$ dispersion can be attributed to one-, two-, or three-photon resonance which occurs through promotion of the most weakly bound outer shell electrons to higher (unfilled) energy states [10]. From our data, the projected $\chi^{(3)}$ resonance wavelength is around $1800 \mathrm{~nm}$, which corresponds to a band energy of $2.07 \mathrm{eV}$. For a three photon process, according to the studies of electronic structure of $\mathrm{Ta}_{2} \mathrm{O}_{5}$ by Arranz et al.[55] this energy transition is only possible for a three-photon resonance involving at least one of the following electronic transitions: $4 f_{7 / 2} \rightarrow 5 d\left(t_{2 g}\right), 4 f_{5 / 2} \rightarrow 5 d\left(t_{2 g}\right), 5 p_{3 / 2} \rightarrow 5 d\left(t_{2 g}\right), 5 p_{1 / 2} \rightarrow 5 d\left(t_{2 g}\right)$.

\section{CONCLUSIONS}

In conclusions, we have developed a complete theoretical and experimental approach for determining the third-order nonlinear susceptibility $\left(\chi^{(3)}\right)$ without the requirement for a second reference material based on measurement and analysis of the frequency tripling effect (i.e. THG), and was applied to a $\mathrm{Ta}_{2} \mathrm{O}_{5}$ waveguide material system. 
We have shown experimentally that THG can be generated over a relatively broad bandwidth $(\sim 140 \mathrm{~nm})$ in our waveguides. The nonlinear coefficient of $\mathrm{Ta}_{2} \mathrm{O}_{5}$ planar waveguides is derived from multi-wavelength analysis of THG outputs. Values for the wavelength range 1470 to $1610 \mathrm{~nm}$ were determined to be from $(7.7 \pm 2.1) \times 10^{-14}$ to $(6.00 \pm 1.26) \times 10^{-13}$ esu with an average value of $(2.673 \pm 1.578) \times 10^{-13}$ esu. The results also give clear indication of $\chi^{(3)}$ dispersion, where $\chi^{(3)}$ values increase with pump wavelength for projected peak at wavelength of $1800 \mathrm{~nm}$. We propose that the $\chi^{(3)}$ enhancement is due to the three-photon resonance that involves the band gap transition of several possible electrons of $\mathrm{Ta}_{2} \mathrm{O}_{5}[10,55]$. The large nonlinear coefficient of $\mathrm{Ta}_{2} \mathrm{O}_{5}$ could be useful for terahertz (THz) spectroscopy[56, 57] and $\mathrm{THz}$ spectroscopy gas sensing[58].

Although applied to measurement of $\chi^{(3)}$ in this paper, our technique and experimental setup is equally suited to measurement of second order susceptibility $\left(\chi^{(2)}\right)$ and analysis of other nonlinear effects, such as the sum-difference frequency generation, SPM, FWM, and cross-phase modulation (XPM).

This technique is suitable for studying and further understanding of the nonlinear effects in $\mathrm{Ta}_{2} \mathrm{O}_{5}$ or other materials (e.g. $\mathrm{Si}, \mathrm{As}_{2} \mathrm{~S}_{3}$, and $\mathrm{TiO}_{2}$ ) based planar devices such as planar waveguides and photonic crystal slabs. The high value of $\chi^{(3)}$ and good optical properties of our Ta2O5 waveguides makes them highly suited for applications as a low spectral noise, wide bandwidth supercontinuum (SC) source. With further optimization of fabrication processes we anticipate that we can reduce the loss to $-4 \mathrm{~dB} / \mathrm{cm}$ in which case nanowire waveguides with a cross-section of $200 \mathrm{~nm}$ by $100 \mathrm{~nm}$ would achieve similar bandwidths from a low cost, small footprint VECSEL[7] pump source.

\section{REFERENCES}

[1] Ishibashi, Y., Orihara, H., Pirc, R. et al., "Dispersion Relation of the Third Order Nonlinear Dielectric Susceptibility in Poly-dispersive System," Journal of the Physical Society of Japan, 73(8), 2323-2325 (2004).

[2] Agrawal, G. P., [Applications of nonlinear fiber optics] Academic Press, San Diego(2001).

[3] Netti, C. M., Zoorob, M. E., Roberts, S. W. et al., "Octave-wide continuum generation in high-index planar waveguide by 1.5-mu $\mathrm{m}$ femtosecond pump." 5714, 195-199.

[4] Lamont, M. R., Luther-Davies, B., Choi, D.-Y. et al., "Supercontinuum generation in dispersion engineered highly nonlinear $(\gamma=10 / \mathrm{W} / \mathrm{m})$ As2S3 chalcogenide planar waveguide," Opt. Express, 16(19), 14938-14944 (2008).

[5] Pelusi, M. D., Ta'eed, V. G., Libin, F. et al., "Applications of Highly-Nonlinear Chalcogenide Glass Devices Tailored for High-Speed All-Optical Signal Processing," Selected Topics in Quantum Electronics, IEEE Journal of, 14(3), 529-539 (2008).

[6] Lamont, M. R., Luther-Davies, B., Choi, D.-Y. et al., "Net-gain from a parametric amplifier on a chalcogenide optical chip," Opt. Express, 16(25), 20374-20381 (2008).

[7] Hoogland, S., Garnache, A., Sagnes, I. et al., "10-GHz train of sub-500-fs optical soliton-like pulses from a surfaceemitting semiconductor laser," Photonics Technology Letters, IEEE, 17(2), 267-269 (2005).

${ }^{[8]}$ Dupriez, P., Finot, C., Malinowski, A. et al., "High-power, high repetition rate picosecond and femtosecond sources based on Yb-doped fiber amplification of VECSELs," Opt. Express, 14(21), 9611-9616 (2006).

[9] Agrawal, G. P., [Nonlinear fiber optics] Elsevier/Academic Press, Amsterdam; London(2007).

[10] Boyd, R. W., [Nonlinear optics] Academic Press, Amsterdam; London, pp. 1-56, 129-144 (2003).

[11] Tai, C.-Y., Wilkinson, J., Perney, N. et al., "Determination of nonlinear refractive index in a Ta2O5 rib waveguide using self-phase modulation," Optics Express, 12(21), 5110-5116 (2004).

[12] Geng, L., and Wright, J. C., "Measurement of the resonant third-order nonlinear susceptibility of C60 by nondegenerate four-wave mixing," Chemical Physics Letters, 249(1-2), 105-111 (1996).

[13] Liao, H. B., Xiao, R. F., Wang, H. et al., "Large third-order optical nonlinearity in Au:TiO[sub 2] composite films measured on a femtosecond time scale," Applied Physics Letters, 72(15), 1817-1819 (1998).

[14] Mohanta, D., and Choudhury, A., "Measurement of third order susceptibility by nonresonant nondegenerate four wave mixing in polymer embedded cadmium sulfide quantum dot systems," Optical Materials, 29(2-3), 342-347 (2006). 
[15] Derkowska, B., Firszt, F., Sahraoui, B. et al., "Study of the third order nonlinear optical properties of Zn1-xMgxSe and Cd1-xMgxSe crystals," Opto-Electronics Review, 16(1), 8-11 (2008).

[16] Sheik-bahae, M., Said, A. A., and Van Stryland, E. W., "High-sensitivity, single-beam n2 measurements," Opt. Lett., 14(17), 955-957 (1989).

[17] Debrus, S., Lafait, J., May, M. et al., "Z-scan determination of the third-order optical nonlinearity of gold:silica nanocomposites," Journal of Applied Physics, 88(8), 4469-4475 (2000).

[18] D'Amore, F., Di Giulio, M., Pietralunga, S. M. et al., "Sputtered stoichiometric TeO[sub 2] glass films: Dispersion of linear and nonlinear optical properties," Journal of Applied Physics, 94(3), 1654-1661 (2003).

[19] Miyano, K., Nishiwaki, T., and Tomioka, A., "A simple and accurate method to determine the third-order nonlinear susceptibility of thin films," Optics Communications, 91(5-6), 501-508 (1992).

[20] Wang, X. H., West, D. P., McKeown, N. B. et al., "Determining the cubic susceptibility (3) of films or glasses by the Maker fringe method: a representative study of spin-coated films of copper phthalocyanine derivation," J. Opt. Soc. Am. B, 15(7), 1895-1903 (1998).

[21] Gubler, U., and Bosshard, C., "Optical third-harmonic generation of fused silica in gas atmosphere: Absolute value of the third-order nonlinear optical susceptibility chi (3)," Physical Review B, 61(16), 10702 (2000).

[22] Meredith, G. R., Buchalter, B., and Hanzlik, C., "Third-order optical susceptibility determination by third harmonic generation. I," The Journal of Chemical Physics, 78(3), 1533-1542 (1983).

[23] Kobayashi, T., Mito, A., Kobayashi, S. et al., "Multiplex method for the measurement of nonlinear susceptibility spectrum applied to third-harmonic generation in a polydiacetylene," Chemical Physics Letters, 433(4-6), 379-384 (2007).

[24] Ghotbi, M., Sun, Z., Majchrowski, A. et al., "Efficient third harmonic generation of microjoule picosecond pulses at $355 \mathrm{~nm}$ in BiB[sub 3]O[sub 6]," Applied Physics Letters, 89(17), 173124-3 (2006).

[25] Stoker, D., Becker, M. F., and Keto, J. W., "Optical third-harmonic generation using ultrashort laser pulses," Physical Review A (Atomic, Molecular, and Optical Physics), 71(6), 061802-4 (2005).

[26] Guillet de Chatellus, H., and Freysz, E., "Measurement of the third-order susceptibility of glasses by EFISH of femtosecond pulses," Optics Express, 9(11), 586-591 (2001).

[27] Zhang, C.-J., Guo, K.-X., and Liang, S.-D., "Third harmonic generation of semiconductor carbon nanotubes," Chemical Physics Letters, 433(1-3), 101-104 (2006).

[28] Banks, P. S., Feit, M. D., and Perry, M. D., "High-intensity third-harmonic generation," Journal of the Optical Society of America B, 19(1), 102-118 (2002).

[29] Hsieh, I. W., Chen, X., Dadap, J. I. et al., "Ultrafast-pulse self-phase modulation and third-order dispersion in Si photonic wire-waveguides," Opt. Express, 14(25), 12380-12387 (2006).

[30] Dadap, J. I., Panoiu, N. C., Chen, X. et al., "Nonlinear-optical phase modification in dispersion-engineered Si photonic wires," Opt. Express, 16(2), 1280-1299 (2008).

[31] Dianov, E. M., Bufetov, I. A., Frolov, A. A. et al., "Catastrophic destruction of fluoride and chalcogenide optical fibres," Electronics Letters, 38(15), 783-784 (2002).

[32] Stegeman, R., Stegeman, G., Delfyett, P. et al., "Raman gain measurements and photo-induced transmission effects of germanium- and arsenic-based chalcogenide glasses," Optics Express, 14(24), 11702-11708 (2006).

[33] Chaneliere, C., Autran, J. L., Devine, R. A. B. et al., "Tantalum pentoxide (Ta2O5) thin films for advanced dielectric applications," Materials Science and Engineering: R: Reports, 22(6), 269-322 (1998).

[34] Florjanczyk, M., Cheben, P., Janz, S. et al., "Multiaperture planar waveguide spectrometer formed by arrayed MachZehnder interferometers," Optics Express, 15(26), 18176-18189 (2007).

[35] Vincent, B., Kremer, R., Boudrioua, A. et al., "Green light generation in a periodically poled Zn-doped LiNbO3 planar waveguide fabricated by He+ implantation," Applied Physics B: Lasers and Optics, 89(2), 235-239 (2007).

[36] Perina, J. J., Haderka, O., Sibilia, C. et al., "Squeezed-light generation in a nonlinear planar waveguide with a periodic corrugation," Physical Review A (Atomic, Molecular, and Optical Physics), 76(3), 033813-14 (2007).

[37] Ayre, M., Cambournac, C., Khayam, O. et al., "Photonic crystal waveguides for coarse-selectivity devices," Photonics and Nanostructures - Fundamentals and Applications, 6(1), 19-25 (2008).

[38] Xiao, L., Cheng, X., and Xu, J., "High-power Nd:YAG planar waveguide laser with YAG and Al2O3 claddings," Optics Communications, 281(14), 3781-3785 (2008).

[39] Fedotova, O., Husakou, A., and Herrmann, J., "Supercontinuum generation in planar rib waveguides enabled by anomalous dispersion," Optics Express, 14(4), 1512-1517 (2006).

[40] Mills, J. D., Chaipiboonwong, T., Brocklesby, W. S. et al., "Observation of the developing optical continuum along a nonlinear waveguide," Opt. Lett., 31(16), 2459-2461 (2006). 
[41] Squier, J., Muller, M., Brakenhoff, G. et al., "Third harmonic generation microscopy," Opt. Express, 3(9), 315-324 (1998).

[42] Yelin, D., and Silberberg, Y., "Laser scanning third-harmonic-generation microscopy in biology," Opt. Express, 5(8), 169-175 (1999).

[43] Mar Blanca, C., and Saloma, C., "Third-Harmonic Generation Microscopy in Highly Scattering Media," Applied Optics, 39(28), 5187-5193 (2000).

[44] Chattopadhyay, A., Mukherjee, S., and Raghuraman, H., "Reverse micellar organization and dynamics: A wavelength-selective fluorescence approach," Journal of Physical Chemistry B, 106(50), 13002-13009 (2002).

[45] Irimpan, L., Krishnan, B., Deepthy, A. et al., "Excitation wavelength dependent fluorescence behaviour of nano colloids of ZnO," Journal of Physics D-Applied Physics, 40(18), 5670-5674 (2007).

[46] Leavesley, S., Jiang, Y., Patsekin, V. et al., "An excitation wavelength--scanning spectral imaging system for preclinical imaging," Review of Scientific Instruments, 79(2), 023707-10 (2008).

[47] Kishida, H., Hasegawa, T., Iwasa, Y. et al., "Dispersion relation in the third-order electric susceptibility for polysilane film," Physical Review Letters, 70(24), 3724-3727 (1993).

[48] Murakami, H., Morita, R., Watanabe, T. et al., "Determination of Third-Order Optical Nonlinearity Dispersion of 1Methyl-1'-Octadecyl-2,2'-Cyanine Perchlorate Langmuir-Blodgett Films Using Electroabsorption Spectroscopy," Japanese Journal of Applied Physics, 39, 5838-5841 (2000).

[49] Okamoto, K., [Fundamentals of optical waveguides] Academic Press, Burlington, Mass.; London(2006).

[50] Eckardt, R. C., Masuda, H., Fan, Y. X. et al., "Absolute and relative nonlinear optical coefficients of KDP, KD*P, $\mathrm{BaB} 2 \mathrm{O} 4, \mathrm{LiIO} 3, \mathrm{MgO}: \mathrm{LiNbO}$, and KTP measured by phase-matched second-harmonic generation,” IEEE Journal of Quantum Electronics, 26(5), 922-933 (1990).

[51] Yariv, A., [Quantum electronics] Wiley, New York(1989).

${ }^{[52]}$ Kaiser, W., [Ultrashort laser pulses and applications] Springer, Berlin(1988).

[53] Jellison, G. E., and Modine, F. A., "Parameterization of the optical functions of amorphous materials in the interband region," Applied Physics Letters, 69(3), 371-373 (1996).

[54] Postava, K., Aoyama, M., Yamaguchi, T. et al., "Spectroellipsometric characterisation of materials for multilayer coatings," Applied Surface Science, 175-176, 276-280 (2001).

[55] Arranz, A., Perez-Dieste, V., and Palacio, C., "Electronic structure of stoichiometric and reduced Ta2O5 surfaces determined by resonant photoemission," Physical Review B, 66(7), 075420 (2002).

[56] Stepanov, A., Kuhl, J., Kozma, I. et al., "Scaling up the energy of THz pulses created by optical rectification," Optics Express, 13(15), 5762-5768 (2005).

[57] Luo, C., Reimann, K., Woerner, M. et al., "Nonlinear terahertz spectroscopy of semiconductor nanostructures," Applied Physics A: Materials Science \& Processing, 78(4), 435-440 (2004).

[58] Mittleman, D. M., Jacobsen, R. H., Neelamani, R. et al., "Gas sensing using terahertz time-domain spectroscopy," Applied Physics B: Lasers and Optics, 67(3), 379-390 (1998). 